Research Article

\title{
When metric and upper dimensions differ in zero divisor graphs of commutative rings
}

\author{
Shane Redmond, Steve Szabo* \\ Department of Mathematics and Statistics, Eastern Kentucky University, Richmond, Kentucky, USA
}

(Received: 12 January 2021. Received in revised form: 15 February 2021. Accepted: 15 February 2021. Published online: 20 February 2021.)

(C) 2021 the authors. This is an open access article under the CC BY (International 4.0) license (www.creativecommons.org/licenses/by/4.0/)

\begin{abstract}
Given a commutative ring $R$, the zero divisor graph of $R$ is the graph $\Gamma(R)$ whose vertex set is the collection of non-zero zero divisors of $R$ and distinct vertices $x$ and $y$ are adjacent precisely when $x y=0$. This article determines the complete list (up to isomorphism) of finite commutative rings $R$ with identity such that the metric dimension and upper dimension of $\Gamma(R)$ differ.
\end{abstract}

Keywords: ring; zero divisor graph; upper dimension.

2020 Mathematics Subject Classification: 13A99, 05C12, $05 \mathrm{C} 78$.

\section{Introduction}

Given a commutative ring $R$ with set of zero divisors $Z(R)$, we define the zero divisor graph of $R$ as the undirected graph $\Gamma(R)$ with vertex set $Z(R)^{*}=Z(R)-\{0\}$ and where distinct vertices $x$ and $y$ are adjacent precisely when $x y=0$. The concept of a zero divisor graph originated with Beck [3] and with Anderson and Naseer [2]. However, this article uses the definition popularized by Anderson and Livingston [1], which uses only the non-zero zero divisors of the ring as vertices of $\Gamma(R)$. Zero divisor graphs are studied to explore the relationship between the algebraic properties of $R$ and the graph properties of $\Gamma(R)$. While many authors have extended this concept to similar graphs on a variety of other algebraic structures, this article will consider only finite commutative rings with $1 \neq 0$. For examples of images of zero divisor graphs, including many of the graphs considered in this article, the reader is referred to [11].

This article serves as a sequel to the paper by Pirzada, Aijaz, and Redmond [10] that explored the metric and upper dimensions of zero divisor graphs of commutative rings. In that article, several classes of rings were shown to have the same metric and upper dimensions, but only one example of a ring was given where the metric and upper dimensions differ. The present paper puts completely to rest the question of which finite rings' zero divisor graphs have differing metric and upper dimensions (see Theorem 5.1).

Section 2 of this article will give the necessary background and results on zero divisor graphs, including definitions of metric dimension, upper dimension, resolving set, and distance similar vertices. Section 3 investigates the graphs of rings which are products of $\mathbb{Z}_{2}$. Section 4 provides further examples of rings which have differing metric and upper dimension. Through the use of distance similar vertices, it will be shown in Section 5 that the examples given in Sections 3 and 4 are indeed the only cases where metric and upper dimensions differ.

\section{Preliminaries}

Definition 2.1. Let $G$ be a finite connected graph, and let $W=\left\{w_{1}, w_{2}, \ldots, w_{k}\right\}$ be an ordered set of vertices of $G$. For any $v \in V(G)$, define the representation $r(v \mid W)$ for $v$ with respect to $W$ as the $k$-tuple

$$
r(v \mid W)=\left(d\left(v, w_{1}\right), d\left(v, w_{2}\right), \ldots, d\left(v, w_{k}\right)\right),
$$

where $d(x, y)$ is the distance between vertices $x$ and $y$ in $G$. The ordered set $W$ is a resolving set if all vertices of $V(G)$ have distinct representations with respect to $W$ and is minimal if no proper subset of $W$ is also a resolving set of $G$.

Keep in mind that the vertices of $W$ clearly have distinct representations as $w_{i}$ is the only vertex of $G$ where $r\left(w_{i} \mid W\right)$ has 0 in the coordinate $i$.

*Corresponding author (steve.szabo@eku.edu). 
Definition 2.2. Let $G$ be a finite connected graph. The cardinality of a minimal resolving set consisting of the smallest number of vertices of $G$ is the metric dimension of $G$, denoted by $\operatorname{dim}(G)$. The cardinality of a minimal resolving set with the largest number of vertices of $G$ is the upper dimension of $G$, denoted by $\operatorname{dim}^{+}(G)$.

Metric dimension of a graph was introduced independently by Slater [12] and by Harary and Melter [5]. Upper dimension of a graph was introduced by Chartrand, Poisson, and Zhang [4]. Clearly, for any connected graph $G$ with $n$ vertices, $\operatorname{dim}(G) \leq \operatorname{dim}^{+}(G) \leq n-1$. For many finite connected graphs, such as complete graphs for example, the upper and metric dimensions are equal. However, it is possible to find finite connected graphs such that the metric and upper dimensions differ. In fact, for every positive integer $N$, there is a finite connected graph $G$ with $\operatorname{dim}^{+}(G) \geq \operatorname{dim}(G)+N$ (see Theorem 2.10 of [4]).

Definition 2.3. Vertices $x$ and $y$ in a graph $G$ are called distance similar if $d(x, a)=d(y, a)$ for all $a \in V(G)-\{x, y\}$.

Previous articles studying resolving sets and metric dimension in zero divisor graphs, such as [6-9], make use of the following important connection between sets of distance similar vertices and minimal resolving sets. (Note, in [7,8], the metric dimension of a graph was called the locating number of the graph.)

Proposition 2.1 (Theorem 2.1, [7]). Let $G$ be a connected graph. Suppose $V(G)$ is partitioned into $k$ distinct distance similar classes $V_{1}, V_{2}, \ldots, V_{k}$ (that is, $x, y \in V_{i}$ if and only if $d(x, a)=d(y, a)$ for all $a \in V(G)-\{x, y\}$ ).

1. Any resolving set $W$ for $G$ contains all but at most one vertex from each $V_{i}$.

2. Each $V_{i}$ induces a complete subgraph or a graph with no edges.

3. $\operatorname{dim}(G) \geq|V(G)|-k$.

4. There exists a minimal resolving set $W$ for $G$ such that if $\left|V_{i}\right|>1$, at most $\left|V_{i}\right|-1$ vertices of $V_{i}$ are elements of $W$.

5. If $m$ is the number of distance similar classes that consist of a single vertex, then $|V(G)|-k \leq \operatorname{dim}(G) \leq|V(G)|-k+m$.

From this point forward, the focus will be on zero divisor graphs of finite commutative rings with identity. Throughout, we denote the ring of integers modulo $n$ by $\mathbb{Z}_{n}$ and the set of non-zero zero divisors of $R$ as $Z(R)^{*}$. The following is one of the most important results about zero divisor graphs and is needed in many results herein. It will be used freely throughout.

Proposition 2.2 (Theorem 2.3, [1]). Let $R$ be a commutative ring. Then $\Gamma(R)$ is connected and diam $(\Gamma(R)) \leq 3$.

For many rings, Proposition 2.1 completely determines the make-up of a resolving set - that is, if $V_{1}, V_{2}, \ldots, V_{k}$ is a partition of $\Gamma(R)$ into distance similar classes and if $y_{i} \in V_{i}$ for $i=1, \ldots, k$, then $W=V_{1} \cup V_{2} \cup \ldots \cup V_{k}-\left\{y_{1}, y_{2}, \ldots, y_{k}\right\}$ is a minimal resolving set. Other rings, like $\mathbb{Z}_{4} \times \mathbb{Z}_{4}$ must include a vertex that is not distance similar to any other vertex in a resolving set (as in the minimal resolving set $\{(1,0),(1,2),(0,1)\}$ ).

Definition 2.4. We call a vertex $v$ in a connected graph $G$ uniquely distance similar if $v$ is not distance similar to any other vertex in $G$. We say a resolving set $W$ for graph $G$ is completely determined by distance similarity if $W$ contains no uniquely distance similar elements.

Lemma 2.1 (Theorem 2.3, [9]). If $G$ is finite connected graph that has a resolving set $W$ that is completely determined by distance similarity, then $\operatorname{dim}(G)=\operatorname{dim}^{+}(G)=|W|$.

\section{Rings that are products of $\mathbb{Z}_{2}$}

In [10], it was shown that for $R \cong \mathbb{Z}_{2} \times \mathbb{Z}_{2} \times \mathbb{Z}_{2} \times \mathbb{Z}_{2}$, $\operatorname{dim}(\Gamma(R)) \neq \operatorname{dim}^{+}(\Gamma(R))$ which was the first such example found. For $R$, both

$$
W=\{(1,1,1,0),(1,1,0,1),(1,0,1,1)\}
$$

and

are minimal resolving sets, so

$$
V=\{(1,0,0,0),(0,1,0,0),(0,0,1,0),(0,0,0,1)\}
$$

$\operatorname{dim}(\Gamma(R)) \leq 3=|W|<|V|=4 \leq \operatorname{dim}^{+}(\Gamma(R))$.

This section culminates with the result that for $n \geq 4$, the metric dimension and upper dimension differ for $\prod_{i=1}^{n} \mathbb{Z}_{2}$. It turns out that these rings are one of only two classes of rings for which metric and upper dimensions differ. The next section will discuss the other class. 
Remark 3.1. When considering rings isomorphic to $\prod_{i=1}^{n} \mathbb{Z}_{2}$, it is worth noting that no two distinct vertices are distance similar when $n \geq 3$.

Throughout this section, for $k, n \in \mathbb{N}$ where $1 \leq k \leq n$, let $W_{k}$ be the set of elements of $\prod_{i=1}^{n} \mathbb{Z}_{2}$ with $k$ non-zero coordinates, and let $w(a)$ be the number of non-zero coordinates of $a \in \prod_{i=1}^{n} \mathbb{Z}_{2}$.

Lemma 3.1. Let $k, n \in \mathbb{N}$ such that $1 \leq k \leq n-1$ and $R=\prod_{i=1}^{n} \mathbb{Z}_{2}$. Then $W_{k}$ is a resolving set for $\Gamma(R)$.

Proof. Let $\overrightarrow{0}=(0, \ldots, 0), \overrightarrow{1}=(1, \ldots, 1)$ and for $x \in R$, let $\vec{x}=\overrightarrow{1}-x$. Also, for $x, y \in R$, define $x \cup y=x+y+x y$ (this is the "componentwise or"). Let $a=\left(a_{1}, \ldots, a_{n}\right), b=\left(b_{1}, \ldots, b_{n}\right) \in V(\Gamma(R))-W_{k}$ with $a \neq b$.

First, assume $w(a) \leq \min \{w(b), n-k\}$. Since $a$ must have at least one 0 where $b$ has a 1 and $a$ has at least $k$ zeros, there exists $v \in W_{k}$ s.t. $a v=\overrightarrow{0}$ and $b v \neq \overrightarrow{0}$. So, $d(a, v)=1 \neq d(b, v)$. The same argument applies when $w(b) \leq \min \{w(a), n-k\}$ by interchanging $a$ and $b$.

Now, assume $w(b) \geq w(a)>n-k$. Since $a$ must have at least one 0 where $b$ has a 1 and $b$ has at most $k-1$ zeros, there exists $v \in W_{k}$ s.t. $a \cup v \neq \overrightarrow{1}$ and $b \cup v=\overrightarrow{1}$. So, $d(a, v)=2 \neq 3=d(b, v)$. In all cases then, $r(a \mid W) \neq r(b \mid W)$. Hence, $W_{k}$ is a resolving set.

Proposition 3.1. Let $n \geq 5, R=\prod_{i=1}^{n} \mathbb{Z}_{2}, 1<k<n-1$ such that $k \neq \frac{n}{2}$ and $w \in W_{k}$. Then $W_{1}, W_{n-1}$ and $W_{k}-\{w\}$ are minimal resolving sets.

Proof. Let $\overrightarrow{0}=(0, \ldots, 0), \overrightarrow{1}=(1, \ldots, 1)$ and for $x \in R$, let $\vec{x}=\overrightarrow{1}-x$. Also, for $x, y \in R$, define $x \cup y=x+y+x y$ (this is the "componentwise or").

By Lemma 3.1, $W_{1}$ and $W_{n-1}$ are resolving sets.

Let $w_{1} \in W_{1}$. Without loss of generality (WLOG) assume $w_{1}=(1,0, \ldots, 0)$. Let $x=(0,1,1,0, \ldots, 0)$ and $y=(1,1,1,0, \ldots, 0)$. Clearly for any $v \in W_{1}-\left\{w_{1}\right\}, d(x, v)=d(y, v)$. Hence, $W_{1}$ is minimal.

Let $w_{n-1} \in W_{n-1}$. WLOG assume $w_{n-1}=(0,1, \ldots, 1)$. Let $x=(0,1,1,0, \ldots, 0)$ and $y=(1,1,1,0, \ldots, 0)$. Since $n>4$, $y \notin W_{n-1}$. Clearly for any $v \in W_{n-1}-\left\{w_{n-1}\right\}, d(x, v)=d(y, v)$. Hence, $W_{n-1}$ is minimal.

Let $W=W_{k}-\{w\}$. Now, we show that $W$ is minimal. Let $z \in W$ and $W^{\prime}=W_{k}-\{w, z\}$. Since $k \neq \frac{n}{2}, \bar{w} \notin W^{\prime}$ and $\bar{z} \notin W^{\prime}$. Then $d(\bar{w}, v)=2=d(\bar{z}, v)$ for all $v \in W^{\prime}$. Hence, $W^{\prime}$ is not a resolving set.

Let $a, b \in V(\Gamma(R))-W$ with $a \neq b$. Assume $a=\bar{w}$. Since $k \neq \frac{n}{2}, a=\bar{w} \notin W$. Then $d(a, v)=2$ for all $v \in W$. Since $b \neq a=\bar{w}$, there exists $v \in W$ s.t. $b v=0$ or $b \cup v=\overrightarrow{1}$ meaning $d(b, v) \in\{1,3\}$ and $d(b, v) \neq d(a, v)$.

For the remainder of the proof assume $a \neq \bar{w}$ and $b \neq \bar{w}$. We proceed in two cases. First, assume $w(a) \leq \min \{w(b), n-k\}$. Since $a \neq \bar{w}$, there exists $v \in W$ s.t. $a v=\overrightarrow{0}$ and $b v \neq \overrightarrow{0}$. So, $d(a, v)=1 \neq d(b, v)$. The same argument applies when $w(b) \leq \min \{w(a), n-k\}$ by interchanging $a$ and $b$.

Now, assume $w(a)>n-k$ and $w(b)>n-k$. Then there exists $v \in W$ s.t. $a \cup v \neq \overrightarrow{1}$ and $b \cup v=\overrightarrow{1}$. So, $d(a, v)=2 \neq 3=d(b, v)$. In all cases then, $r(a \mid W) \neq r(b \mid W)$. Hence, $W$ is a minimal resolving set.

Theorem 3.1. Let $n \geq 2$ and $R=\prod_{i=1}^{n} \mathbb{Z}_{2}$. Then $\operatorname{dim}(R) \neq \operatorname{dim}^{+}(R)$ if and only if $n \geq 4$.

Proof. As seen in [10], the metric and upper dimensions of are equal when $n \in\{2,3\}$ (these cases can easily be checked by hand). When $n=4$, the metric and upper dimensions of are not equal. This was given at the beginning of this section. Finally, assume $n \geq 5$. Proposition 3.1 shows that there is a minimal resolving set of size $n$ and also another minimal resolving set of size $\left(\begin{array}{l}n \\ 2\end{array}\right)-1$. So,

$$
\operatorname{dim}(R) \leq n<\left(\begin{array}{l}
n \\
2
\end{array}\right)-1 \leq \operatorname{dim}^{+}(R)
$$

\section{Other examples where metric and upper dimensions differ}

In the last section, it was shown that for products of at least 4 copies of $\mathbb{Z}_{2}$, the metric and upper dimensions of the zero divisor graph differ. Here the only other rings with this property are provided. These exceptions are

$$
S \times \prod_{i=1}^{n} \mathbb{Z}_{2}
$$

where $n \geq 3$ and $S \in\left\{\mathbb{Z}_{4}, \mathbb{Z}_{2}[X] /\left(X^{2}\right)\right\}$. 
Example 4.1. It can be easily verified that

$$
\operatorname{dim}\left(\Gamma\left(\mathbb{Z}_{4} \times \mathbb{Z}_{2}\right)\right)=\operatorname{dim}^{+}\left(\Gamma\left(\mathbb{Z}_{4} \times \mathbb{Z}_{2}\right)\right)=2 \quad \text { and } \quad \operatorname{dim}\left(\Gamma\left(\mathbb{Z}_{4} \times \mathbb{Z}_{2} \times \mathbb{Z}_{2}\right)\right)=\operatorname{dim}^{+}\left(\Gamma\left(\mathbb{Z}_{4} \times \mathbb{Z}_{2} \times \mathbb{Z}_{2}\right)\right)=4 .
$$

However, in $\mathbb{Z}_{4} \times \prod_{i=1}^{3} \mathbb{Z}_{2}$,

$$
\begin{array}{r}
\{(1,0,0,0),(1,0,0,1),(1,0,1,0),(1,0,1,1), \\
(1,1,0,0),(1,1,0,1),(1,1,1,0),(2,0,0,0)\}
\end{array}
$$

and

$$
\begin{array}{r}
\{(1,0,0,0),(1,0,0,1),(1,0,1,0),(1,0,1,1),(1,1,0,0), \\
(1,1,0,1),(1,1,1,0),(2,0,0,1),(2,0,1,0),(2,1,0,0)\}
\end{array}
$$

are minimal resolving sets which shows

$$
\operatorname{dim}\left(\Gamma\left(\mathbb{Z}_{4} \times \mathbb{Z}_{2} \times \mathbb{Z}_{2} \times \mathbb{Z}_{2}\right)\right) \neq \operatorname{dim}^{+}\left(\Gamma\left(\mathbb{Z}_{4} \times \mathbb{Z}_{2} \times \mathbb{Z}_{2} \times \mathbb{Z}_{2}\right)\right)
$$

Replacing $\mathbb{Z}_{4}$ with $\mathbb{Z}_{2}[X] /\left(X^{2}\right)$, similar results hold.

Theorem 4.1. Let

$$
R=S \times \prod_{i=1}^{k} \mathbb{Z}_{2}
$$

where $k \geq 3$ and $S \in\left\{\mathbb{Z}_{4}, \mathbb{Z}_{2}[X] /\left(X^{2}\right)\right\}$. Then the resolving sets of $\Gamma(R)$ are not completely determined by distance similarity, $\operatorname{dim}(\Gamma(R))=2^{k}$ and $\operatorname{dim}^{+}(\Gamma(R)) \geq 2^{k}+k-1$.

Proof. The proof is written for $S=\mathbb{Z}_{4}$, but the same arguments apply for $S=\mathbb{Z}_{2}[X] /\left(X^{2}\right)$ if " 3 " is replaced with " $1+X$ " and "2" is replaced with " $\mathrm{X}$ ".

Let $R=\mathbb{Z}_{4} \times \prod_{i=1}^{k} \mathbb{Z}_{2}$. Then the only vertices distance similar to some other vertex of $\Gamma(R)$ come in pairs of the from $\left(1, r_{1}, \ldots, r_{k}\right)$ and $\left(3, r_{1}, \ldots, r_{k}\right)$. For any vertex $v$ of this from, note $d(v,(2,0, \ldots, 0))=d(v,(1,0, \ldots, 0))=d(v,(3,0, \ldots, 0))=$ 2. Thus, letting $W$ be any collection of vertices consisting of only one of each pair of distance similar vertices, $W$ could not be a minimal resolving set as $(2,0, \ldots, 0) \notin W$ and at most one of $(1,0, \ldots, 0)$ and $(3,0, \ldots, 0)$ would be in $W$. Hence, a minimal resolving set must contain some uniquely distance similar vertex.

Define $T=\left\{v=\left(v_{1}, \ldots, v_{k+1}\right) \mid v_{1}=1, v_{i}=0\right.$ for at least one $\left.i \geq 2\right\}$. Then $T$ consists of one element of each pair of distance similar vertices and $|T|=2^{k}-1$. Let $T^{*}=T \cup\{(2,0, \ldots, 0)\}$. In light of the above paragraph, if $T^{*}$ is a resolving set, then it is the smallest possible minimal resolving set. Let $x=\left(x_{1}, \ldots, x_{k+1}\right), y=\left(y_{1}, \ldots, y_{k+1}\right) \in V(\Gamma(R))-T^{*}$. If, WLOG, $x_{j}=1$ and $y_{j}=0$ for some $j \geq 2$, then, letting $w$ be the element of $T^{*}$ with 0 in coordinate $j$ and 1 in all other coordinates, $d(x, w)=1$ or 3 but $d(y, w)=2$. If, WLOG, $x_{1}=0$ and $y_{1} \neq 0$, then $(1,0, \ldots, 0) \in T^{*}$ and $d(x,(1,0, \ldots, 0))=1$ but $d(y,(1,0, \ldots, 0))>1$. If, WLOG, $x_{1}=2$ and $y_{1}=3$, then $d(x,(2,0, \ldots, 0))=1$ and $d(y,(2,0, \ldots, 0))>1$. Hence, $T^{*}$ is a minimal resolving set and $\operatorname{dim}(\Gamma(R))=\left|T^{*}\right|=2^{k}$ (this is also true for $k \in\{1,2\}$ ).

Remember $k \geq 3$. Define $T$ as above. Define $Q=\left\{v=\left(v_{1}, \ldots, v_{k+1}\right) \mid v_{1}=2, v_{i}=1\right.$ for only one $\left.i \geq 2\right\}$ and let $U=T \cup Q$. We will show $U$ is a minimal resolving set. Let $x=\left(x_{1}, \ldots, x_{k+1}\right), y=\left(y_{1}, \ldots, y_{k+1}\right) \in V(\Gamma(R))-U$. The cases where, WLOG, $x_{j}=1$ and $y_{j}=0$ for $j \geq 2$ or, WLOG, $x_{1}=0$ and $y_{1} \neq 0$ hold as in the above paragraph. So, suppose, WLOG, $x_{1}=2, y_{1}=3$, and $x_{j}=y_{j}$ for each $j \geq 2$. Some coordinate of $y$ must be zero, so say WLOG, $y_{2}=x_{2}=0$. Then $(2,1,0, \ldots, 0) \in U$ with $d(x,(2,1,0, \ldots, 0))=1$ but $d(y,(2,1,0, \ldots, 0))>1$. Thus $U$ is a resolving set.

Next, we show $U$ is minimal. Note that by Proposition 2.1, if any subset $M$ of $U$ is a resolving set $T \subseteq M$. Suppose $M$ does not contain some element of $Q$. WLOG, consider the case where $M=U-\{(2,1,0, \ldots, 0)\}$. For $x^{*}=(2,0,1, \ldots, 1)$ and $y^{*}=(3,0,1, \ldots, 1)$, note $x^{*}, y^{*} \in V(\Gamma(R))-U$. For any element $q \in Q-\{(2,1,0, \ldots, 0)\}, d\left(x^{*}, q\right)=d\left(y^{*}, q\right)=2$. For any $t=\left(t_{1}, \ldots, t_{k+1}\right) \in T, d\left(x^{*}, t\right)=d\left(y^{*}, t\right)=2$ if $t_{2}=0$ or $d\left(x^{*}, t\right)=d\left(y^{*}, t\right)=3$ if $t_{2}=1$. Hence, $r\left(x^{*} \mid M\right)=r\left(y^{*} \mid M\right)$. Thus, $U$ is a minimal resolving set. Therefore $\operatorname{dim}^{+}(\Gamma(R)) \geq|U|=\left(2^{k}-1\right)+k$.

Corollary 4.1. Let

$$
R=S \times \prod_{i=1}^{k} \mathbb{Z}_{2}
$$

where $k \geq 3$ and $S \in\left\{\mathbb{Z}_{4}, \mathbb{Z}_{2}[X] /\left(X^{2}\right)\right\}$. Then $\operatorname{dim}(\Gamma(R)) \neq \operatorname{dim}^{+}(\Gamma(R))$. 


\section{When metric and upper dimension of $\Gamma(R)$ are equal}

In the previous sections, examples were given of rings whose metric and upper dimension differ. In this final section, it will be shown that those examples are the only rings with that property giving a complete characterization of such rings.

Theorem 5.1. Let $R$ be a finite commutative ring that is not a field. Then $\operatorname{dim}(\Gamma(R)) \neq \operatorname{dim}^{+}(\Gamma(R))$ if and only if

$$
R \cong S \times \prod_{i=1}^{m} \mathbb{Z}_{2}
$$

where $m \geq 3$ and $S \in\left\{\mathbb{Z}_{2}, \mathbb{Z}_{4}, \mathbb{Z}_{2}[X] /\left(X^{2}\right)\right\}$.

The proof of Theorem 5.1 will be given at the end of the section. We first must characterize the finite rings where the resolving sets are completely determined by distance similar vertices.

Lemma 5.1. Let $R$ be a finite commutative local ring with identity that is not a field.

(i) $\Gamma(R)$ does not have at least two distinct vertices that are distance similar if and only if $R$ is isomorphic to $\mathbb{Z}_{4}$ or $\mathbb{Z}_{2}[X] /\left(X^{2}\right)$,

(ii) If $\Gamma(R)$ has a vertex $x$ that is uniquely distance similar, then $2 x=0$ and $x$ is the only vertex such that $x z=0$ for all $z \in V(\Gamma(R))$.

Proof. (i) Let $x \in V(\Gamma(R))=Z(R)^{*}$. Since $R$ is local, there is some $a \in Z(R)^{*}$ such that $a z=0$ for all $z \in Z(R)$. If $x+a \neq 0$, then $x+a \neq x$ and $x+a \neq a$, and $x$ and $x+a$ are distance similar vertices (since $z x=0$ if and only if $z(x+a)=0$ ). If $x+a=0$, then $x=-a$. Therefore, $x$ and $a$ are distance similar if $x \neq a$. The only cases where $Z(R)^{*}=\{a\}$ are $\mathbb{Z}_{4}$ or $\mathbb{Z}_{2}[X] /\left(X^{2}\right)$.

(ii) If $x$ is uniquely distance similar, then $2 x=0$, for otherwise $x$ and $-x$ would be distance similar. If there was any vertex $a \neq x$ such that $a z=0$ for all $z \in Z(R)$, the $x$ and $x+a$ would be distance similar. Since $R$ is local implies there is some $t \in Z(R)^{*}$ with $t Z(R)=\{0\}$, it must be the case that $x Z(R)=\{0\}$.

Note, that if $R$ is isomorphic to $\mathbb{Z}_{4}$ or $\mathbb{Z}_{2}[X] /\left(X^{2}\right)$, then $\operatorname{dim}(\Gamma(R))=\operatorname{dim}^{+}(\Gamma(R))$ as there is only one vertex in $\Gamma(R)$. Thus, we will treat these two rings as special cases in the following results.

Proposition 5.1. Let $R$ be a finite commutative local ring with identity that is not a field and not isomorphic to $\mathbb{Z}_{4}$ or $\mathbb{Z}_{2}[X] /\left(X^{2}\right)$. Then $\Gamma(R)$ has a resolving set completely determined by distance similarity.

Proof. If $\Gamma(R)$ has no uniquely distance similar vertices, then the result is clearly true by Proposition 2.1. So, suppose $x$ is uniquely distance similar in $\Gamma(R)$. Then, by Lemma 5.1, $x$ is the only vertex such that $x z=0$ for all $z \in V(\Gamma(R))$. Let $V_{1}, V_{2}, \ldots, V_{k}$ be a partition of $\Gamma(R)$ into distance similar classes. Let $y_{i} \in V_{i}$ for $i=1, \ldots, k$ and $W=V_{1} \cup V_{2} \cup$ $\ldots \cup V_{k}-\left\{y_{1}, y_{2}, \ldots, y_{k}\right\}$. All that remains to verify $W$ is a minimal resolving set is to verify $r(x \mid W) \neq r(a \mid W)$ for all $a \in V(\Gamma(R))-(W \cup\{x\})$. However, if $a \in V(\Gamma(R))-(W \cup\{x\})$, there is some $t \in V(\Gamma(R))$ with $t a \neq 0$. Note that $t$ is distance similar to $t+x$, so either $t \in W$ or $t+x \in W$ with $d(a, t+x)=d(a, t) \neq 1$. However, $d(x, w)=1$ for all $w \in W$.

Now, we will use the fact that any finite commutative ring with identity can be written as a direct product of local rings.

Theorem 5.2. Let $n \geq 1$ and $R=\prod_{1}^{n} R_{i}$ where each $R_{i}$ is a finite commutative local ring with identity and if $n=1, R_{1}$ is not a field and not isomorphic to $\mathbb{Z}_{4}$ or $\mathbb{Z}_{2}[X] /\left(X^{2}\right)$. Then $R$ has a resolving set completely determined by distance similarity if and only if $R$ is not isomorphic to any ring of the form

$$
S \times \prod_{i=1}^{k} \mathbb{Z}_{2}
$$

where $k \geq 1$ and $S \in\left\{\mathbb{Z}_{2}, \mathbb{Z}_{4}, \mathbb{Z}_{2}[X] /\left(X^{2}\right)\right\}$.

Proof. If $n=1$, by Proposition 5.1, the result holds. Assume $n \geq 2$. If $\Gamma(R)$ has no uniquely distance similar vertices, then the result is clearly true by Proposition 2.1. So, suppose $x=\left(x_{1}, \ldots, x_{n}\right)$ is uniquely distance similar in $\Gamma(R)$. If $x_{i}$ is distance similar to $r_{i}$ in some $\Gamma\left(R_{i}\right)$, then $r=\left(x_{1}, \ldots, x_{i-1}, r_{i}, x_{i+1}, \ldots, x_{n}\right)$ is distance similar to $x$ in $\Gamma(R)\left(\operatorname{as} x\left(a_{1}, \ldots, a_{n}\right)=(0, \ldots, 0)\right.$ if and only if $r\left(a_{1}, \ldots, a_{n}\right)=(0, \ldots, 0)$ for any $\left.\left(a_{1}, \ldots, a_{n}\right) \in Z(R)\right)$. Thus, this leaves only three possibilities for each $x_{i}$ :

Case A: $x_{i}=0$. 
Case B: $x_{i} \in Z\left(R_{i}\right)^{*}$. Since $R_{i}$ is local, there is some $a_{i} \in Z\left(R_{i}\right)$ such that $a_{i} Z\left(R_{i}\right)=\{0\}$. However, this would mean, as in Lemma 5.1, $x$ is distance similar to $\left(x_{1}, \ldots, x_{i-1}, x_{i}+a_{i}, x_{i+1}, \ldots, x_{n}\right)$ unless $x_{i}=a_{i}$ and $x_{i}$ is the only element of $Z\left(R_{i}\right)^{*}$ such that $x_{i} Z(R)=\{0\}$.

Case C: $x_{i} \in R_{i}-Z\left(R_{i}\right)$. If $R_{i} \not \mathbb{Z}_{2}$, then $\left|R_{i}-Z\left(R_{i}\right)\right| \geq 2$. If $t_{i} \in R_{i}-Z\left(R_{i}\right)$ with $t_{i} \neq x_{i}$, then $x$ is distance similar to $\left(x_{1}, \ldots, x_{i-1}, t_{i}, x_{i+1}, \ldots, x_{n}\right)$. Hence, if $x_{i} \in R_{i}-Z\left(R_{i}\right)$, then $R_{i} \simeq \mathbb{Z}_{2}$ and $x_{i}=1$.

Let $V_{1}, V_{2}, \ldots, V_{k}$ be a partition of $\Gamma(R)$ into distance similar classes. Let $v_{i} \in V_{i}$ for $i=1, \ldots, k$ and $W=V_{1} \cup V_{2} \cup \ldots \cup$ $V_{k}-\left\{v_{1}, v_{2}, \ldots, v_{k}\right\}$. Let $y=\left(y_{1}, \ldots, y_{n}\right) \in V(\Gamma(R))-(W \cup\{x\})$.

Case 1: There is some coordinate $i$ with $x_{i}=0$ and $y_{i} \neq 0$. If $R_{i} \not \mathbb{Z}_{2}$, then choose distinct elements $t_{i}, t_{i}^{*} \in R_{i}-Z\left(R_{i}\right)$ and define $t=\left(0, \ldots, 0, t_{i}, 0, \ldots, 0\right)$ and $t^{*}=\left(0, \ldots, 0, t_{i}^{*}, 0, \ldots, 0\right)$. Then either $t \in W$ or $t^{*} \in W$ with $d(x, t)=d\left(x, t^{*}\right)=1$ and $d(y, t)=d\left(y, t^{*}\right)>1$.

So, suppose $R_{i} \simeq \mathbb{Z}_{2}$ with $x_{i}=0$ and $y_{i}=1$. Then define $t=(1, \ldots, 1,0,1, \ldots, 1)$ with $t_{i}=0$. Note $d(x, t)=2$ but $d(y, t) \in\{1,3\}$. Also, unless each $R_{j} \simeq \mathbb{Z}_{2}$, either $t \in W$ or some distance similar vertex $t^{*} \in W$ (as replacing 1 with any element of $R_{j}-Z\left(R_{j}\right)$ in coordinate $j$ produces an element distance similar to $t$ if $\left.R_{j} \not \mathbb{Z}_{2}\right)$.

Case 2: There is some coordinate $i$ with $y_{i}=0$ and $x_{i} \neq 0$. By an argument analogous to the last case, we can find $t \in W$ with $d(y, t) \neq d(x, t)$ unless each $R_{j} \simeq \mathbb{Z}_{2}$.

Case 3: There is some coordinate $i$ with $x_{i} \in Z\left(R_{i}\right)^{*}$. Then, as in the above proposition, $x_{i} Z\left(R_{i}\right)=\{0\}$. As seen in Case 2 , we can assume $y_{i} \neq 0$. Suppose $R_{i} \nsucceq \mathbb{Z}_{4}$ and $R_{i} \nsucceq \mathbb{Z}_{2}[X] /\left(X^{2}\right)$. If $y_{i} \notin Z(R)^{*}$, then, by Lemma 5.1 , we can choose distinct elements $t_{i}$ and $t_{i}^{*}$ of $Z\left(R_{i}\right)^{*}$ that are distance similar in $\Gamma\left(R_{i}\right)$. Define $t=\left(0, \ldots, 0, t_{i}, 0, \ldots, 0\right)$ and $t^{*}=\left(0, \ldots, 0, t_{i}^{*}, 0, \ldots, 0\right)$. Then either $t \in W$ or $t^{*} \in W$ with $d(x, t)=d\left(x, t^{*}\right)=1$ and $d(y, t)=d\left(y, t^{*}\right)>1$. If instead $y_{i} \in Z(R)^{*}-\left\{x_{i}\right\}$, then there is some $q_{i} \in Z\left(R_{i}\right)$ with $y_{i} q_{i} \neq 0$. Let $q=\left(0, \ldots, 0, q_{i}, 0, \ldots, 0\right)$ and $q^{*}=\left(0, \ldots, 0, q_{i}+x_{i}, 0, \ldots, 0\right)$. Then $q$ and $q^{*}$ are distance similar in $\Gamma(R)$ and therefore either $q \in W$ or $q^{*} \in W$. Also, $d(x, q)=d\left(x, q^{*}\right)=1$ but $d(y, q)=d\left(y, q^{*}\right)>1$. Hence, $r(x \mid W) \neq r(y \mid W)$ unless $x_{i}=y_{i}$.

Now, let us assume $r(x \mid W)=r(y \mid W)$ and $R \not \prod_{i}^{n} \mathbb{Z}_{2}$. Since $x$ is a zero divisor, some coordinate of $x_{i}$ must be 0 or a zero divisor of $R_{i}$.

If $x_{i}=0$, then $y_{i}=0$ by Case 1 .

If $x_{i}=1$, then $R_{i} \simeq \mathbb{Z}_{2}$ and, by Case $2, y_{i}=1=x_{i}$.

If $x_{i} \in Z\left(R_{i}\right)^{*}$, then, as in Case 3 , either $x_{i}=y_{i}$ or $R_{i} \simeq \mathbb{Z}_{4}$ or $\mathbb{Z}_{2}[X] /\left(X^{2}\right)$.

Since $x \neq y$, it must be the case that $R_{i} \simeq \mathbb{Z}_{4}$ or $\mathbb{Z}_{2}[X] /\left(X^{2}\right)$ with $x_{i} \in Z\left(R_{i}\right)^{*}$ and $y_{i} \in R_{i}-Z\left(R_{i}\right)$ for some $i$.

Suppose for any $k \neq i, R_{k}$ is not isomorphic to $\mathbb{Z}_{2}, \mathbb{Z}_{4}$ or $\mathbb{Z}_{2}[X] /\left(X^{2}\right)$. Note that this would imply $x_{k} \neq 1$. If $x_{k}=0$, choose distinct $t_{k}, t_{k}^{*} \in R_{k}-Z\left(R_{k}\right)$. Define $t$ to be the element of $R$ with $t_{k}$ in coordinate $k, x_{i}$ in coordinate $i$, and 0 in all other coordinates. Similarly, define $t^{*}$ to be the element of $R$ with $t_{k}^{*}$ in coordinate $k, x_{i}$ in coordinate $i$, and 0 in all other coordinates. Then either $t \in W$ or $t^{*} \in W$ with $d(x, t)=d\left(x, t^{*}\right)=1$ and $d(y, t)-d\left(y, t^{*}\right)>1$. If $x_{k} \in Z\left(R_{k}\right)^{*}$, then, by the above proposition, we can choose distinct $a_{k}, b_{k} \in Z\left(R_{k}\right)^{*}$ such that $a_{k}$ and $b_{k}$ are distance similar in $\Gamma\left(R_{k}\right)$. Define $a$ to be the element of $R$ with $a_{k}$ in coordinate $k, x_{i}$ in coordinate $i$, and 0 in all other coordinates. Similarly, define $b$ to be the element of $R$ with $b_{k}$ in coordinate $k, x_{i}$ in coordinate $i$, and 0 in all other coordinates. Then either $a \in W$ or $b \in W$ with $d(a, x)=d(b, x)=1$ but $d(a, y)=d(b, y)>1$.

Hence, having reached a contradiction to $r(x \mid W)=r(y \mid W)$ in all possible cases, it must be the case that $W$ is a minimal resolving set except possibly in the cases where each $R_{i}$ is isomorphic to $\mathbb{Z}_{2}$ or $\mathbb{Z}_{4}$ or $\mathbb{Z}_{2}[X] /\left(X^{2}\right)$.

Finally, suppose each $R_{i}$ is isomorphic to $\mathbb{Z}_{2}, \mathbb{Z}_{4}$ or $\mathbb{Z}_{2}[X] /\left(X^{2}\right)$ where $\left|R_{j}\right|>2$ and $\left|R_{k}\right|>2$ for some $j \neq k$. That is, WLOG, say $R_{1} \simeq \mathbb{Z}_{4}$ or $\mathbb{Z}_{2}[X] /\left(X^{2}\right)$, and $R_{2} \simeq \mathbb{Z}_{4}$ or $\mathbb{Z}_{2}[X] /\left(X^{2}\right)$. Define $W, x$, and $y$ as above. Suppose $r(x \mid W)=r(y \mid W)$. As seen above, if there is some coordinate $i$ where $x_{i} \neq y_{i}$, then $R_{i} \neq \mathbb{Z}_{2}$. Thus, $x$ and $y$ differ only in coordinates $j$ where $R_{j} \simeq \mathbb{Z}_{4}$ or $\mathbb{Z}_{2}[X] /\left(X^{2}\right)$. Also as seen above, it cannot be the case that $x_{j}=0$ or $y_{j}=0$ in these coordinates.

So, since $x \neq y$, WLOG say $x_{1} \neq y_{1}$. Then, as above, it must be the case $x_{1} \in Z\left(R_{1}\right)^{*}$ and $y_{1} \in R_{1}-Z\left(R_{1}\right)$. Let $s_{1}, s_{2} \in R_{2}-Z\left(R_{2}\right)$ with $s_{1} \neq s_{2}$, and let $t=\left(x_{1}, s_{1}, 1, \ldots, 1\right)$ and $t^{*}=\left(x_{1}, s_{2}, 1, \ldots, 1\right)$. Then $t$ and $t^{*}$ are distance similar, implying $t \in W$ or $t^{*} \in W$. Note $d(x, t)=d\left(x, t^{*}\right)=2$, but $d(y, t)=d\left(y, t^{*}\right)=3$. This contradicts that $r(x \mid W)=r(y \mid W)$, implying $W$ is a minimal resolving set.

Proof of Theorem 5.1. If $R$ is not of the form given then, by Theorem 5.2 and Lemma $2.1, \operatorname{dim}(\Gamma(R))=\operatorname{dim}+(\Gamma(R))$. Now, assume

$$
R \cong S \times \prod_{i=1}^{k} \mathbb{Z}_{2}
$$

where $k \geq 1$ and $S \in\left\{\mathbb{Z}_{2}, \mathbb{Z}_{4}, \mathbb{Z}_{2}[X] /\left(X^{2}\right)\right\}$. Example 4.1 shows that when $1 \leq k \leq 2$ and $S \in\left\{\mathbb{Z}_{4}, \mathbb{Z}_{2}[X] /\left(X^{2}\right)\right\}, \operatorname{dim}(\Gamma(R))=$ $\operatorname{dim}^{+}(\Gamma(R))$. Theorem 3.1 shows that when $1 \leq k \leq 2$ and $S=\mathbb{Z}_{2}$, $\operatorname{dim}(\Gamma(R))=\operatorname{dim}^{+}(\Gamma(R))$. Finally, if $k \geq 3$, Proposition 3.1 and Corollary 4.1 show that $\operatorname{dim}(\Gamma(R)) \neq \operatorname{dim}^{+}(\Gamma(R))$. 


\section{References}

[1] D. F. Anderson, P. S. Livingston, The zero-divisor graph of a commutative ring, J. Algebra 217 (1999) 434-447.

[2] D. D. Anderson, M. Naseer, Beck's coloring of a commutative ring, J. Algebra 159 (1993) 500-514.

[3] I. Beck, Coloring of commutative rings, J. Algebra 116 (1988) 208-226.

[4] G. Chartrand, C. Poisson, P. Zhang, Resolvability and the upper dimension of graphs, Comput. Math. Appl. 39 (2000) 19-28.

[5] F. Harary, R. A. Melter, On the metric dimension of a graph, Ars Combin. 2 (1976) 191-195.

[6] S. Pirzada, M. Aijaz, Metric and uppder dimension of zero divisor graphs associated to commutative rings, Acta Univ. Sapientiae Inform. 12 (2020) 84-101.

[7] S. Pirzada, R. Raja, S. P. Redmond, Locating sets and numbers of graphs associated to commutative rings, J. Algebra Appl. 13 (2014) Art\# 1450047.

[8] R. Raja, S. Pirzada, S. P. Redmond, On locating numbers and codes of zero divisor graphs associated with commutative rings, J. Algebra Appl. 15 (2016) Art\# 1650014.

[9] S. Pirzada, M. Aijaz, S. P. Redmond, On upper dimension of graphs and their bases sets, Discrete Math. Lett. 3 (2020) 37-43.

[10] S. Pirzada, M. Aijaz, S. P. Redmond, Upper dimension and bases of zero-divisor graphs of commutative rings, AKCE Int. J. Graphs Comb. 17 (2020) $168-173$.

[11] S. P. Redmond, On zero-divisor graphs of small finite commutative rings, Discrete Math. 307 (2007) 1155-1166.

[12] P. J. Slater, Leaves of trees, Congr. Numer. 14 (1975) 549-559. 